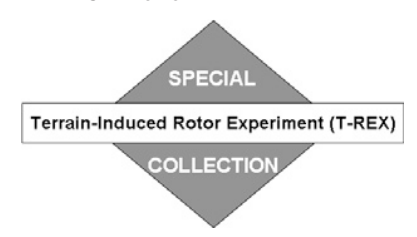

\title{
Coplanar Doppler Lidar Retrieval of Rotors from T-REX
}

\author{
Michael Hill, Ron Calhoun, and H. J. S. Fernando \\ Department of Mechanical and Aerospace Engineering, Arizona State University, Tempe, Arizona \\ ANDREAS WIESER \\ Institut für Meteorologie und Klimaforschung, Forschungszentrum Karlsruhe, Germany \\ ANDREAS DÖRNBRACK AND MARTIN WEISSMANN \\ Deutsches Zentrum für Luft- und Raumfahrt, Institut für Physik der Atmosphäre, Oberpfaffenhofen, Germany \\ GEORG MAYR \\ Institute of Meteorology and Geophysics, University of Innsbruck, Austria \\ ROBERT NEWSOM \\ Pacific Northwest National Laboratory, Richland, Washington
}

(Manuscript received 20 November 2008, in final form 5 June 2009)

\begin{abstract}
Dual-Doppler analysis of data from two coherent lidars during the Terrain-Induced Rotor Experiment (T-REX) allows the retrieval of flow structures, such as vortices, during mountain-wave events. The spatial and temporal resolution of this approach is sufficient to identify and track vortical motions on an elevated, cross-barrier plane in clear air. Assimilation routines or additional constraints such as two-dimensional continuity are not required. A relatively simple and quick least squares method forms the basis of the retrieval. Vortices are shown to evolve and advect in the flow field, allowing analysis of their behavior in the mountain-wave-boundary layer system. The locations, magnitudes, and evolution of the vortices can be studied through calculated fields of velocity, vorticity, streamlines, and swirl. Generally, observations suggest two classes of vortical motions: rotors and small-scale vortical structures. These two structures differ in scale and behavior. The level of coordination of the two lidars and the nature of the output (i.e., in range gates) creates inherent restrictions on the spatial and temporal resolution of retrieved fields.
\end{abstract}

\section{Introduction}

Rotary flows in the lee of mountains are thought to be associated with enhanced mechanical shear caused or accentuated by complex mountain flows such as largescale separation, recirculation, and formation of lee waves (Doyle and Durran 2002). Lee waves create adverse pressure gradients, which may lead to reverse or recirculating flow near the ground. Interest in rotary flows near topography has been documented as far back

Corresponding author address: Ron Calhoun, Mechanical and Aerospace Engineering, Arizona State University, Main Campus, P.O. Box 876106, Tempe, AZ 85287-6106.

E-mail: ron.calhoun@asu.edu as the 1880s when Mohorovičić noted a "permanent" cloud mass near Bakar Bay, Croatia, that was likely a result of a large circulating wind (Grubišić and Orlić 2007). Observational evidence of these flows has been plentiful, including, for example, the movement of dust plumes lofted by strong winds, encounters of severe turbulence from aircraft, ground-based atmospheric measurements, and rotor or mountain-wave clouds visible from both the ground and from space. Recently, numerical simulation has revealed detailed vortical motions embedded in larger-scale flows in the lee of mountains (Doyle and Durran 2007). These simulations are helping to illuminate the importance of surface friction in rotor development by showing, for example, that rotors can fail to develop, even in instances of high shear, if the 


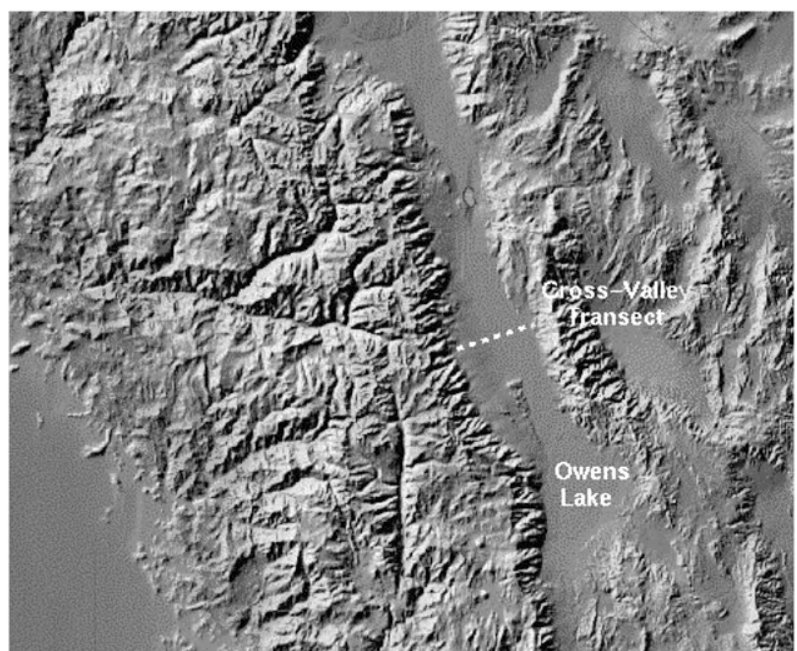

FIG. 1. Topography of Owens Valley, California. Location of Cross Valley transect for T-REX (Grubišić et al. 2004).

atmospheric state is unfavorable for lee wave formation. In this paper, we show that two coherent Doppler lidar scanning the same vertical-horizontal plane can provide direct observational evidence showing the spatial extent, strength, and behavior of vortical flows in clear air below cloud level.

The two Doppler lidar performed simultaneous rangeheight indicator (RHI) scans with the same azimuthal angle, creating a cross-barrier plane on which two sets of radial velocities were measured. Both lidars' RHI scans provided portions of wind velocity measured along the respective beam as a function of range and elevation over $180^{\circ}$ in the constant azimuthal plane every $30 \mathrm{~s}$. (Figure 1 shows a transect of the "cross-barrier" direction; Fig. 2 shows the relative locations of the lidars and their ranges on the plane of interest.) This allowed velocity vectors to be retrieved using the least squares algorithm described below. The scanned plane was well positioned to sample vortices with horizontal axes parallel to the north-south mountain range; that is, an expected orientation for vortices formed due to topographically induced shear layers (see Calhoun and Street 2001; Calhoun et al. 2001).

The retrieved two-dimensional velocity fields allow the calculation of vorticity, streamlines, and swirl on the cross-barrier plane. The vortical motions on the crossbarrier plane can be classified roughly in two categories: 1) larger-scale, less coherent vortical motions linked to and energized by velocity differences between westerly flow aloft and a low-level return flow (rotors, in our terminology below), and 2) stronger, more coherent vortices of more limited spatial extent, which typically advect with the westerly flow aloft but may be entrained into recirculating flow nearer the ground.

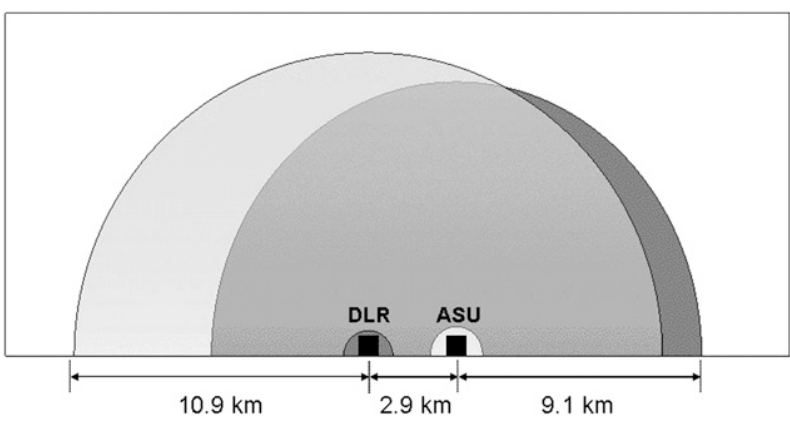

FIG. 2. Radial overlap of coplane lidar scanning in $80^{\circ}$ azimuth (not to scale).

\section{Background}

\section{a. Relevance of mountain flows}

Air pollution and aircraft safety motivate the study of vortical flows in the lee of mountains. For example, predicting air quality in Owens Valley, California, hinges on understanding rotor development and its strong association with low-level wind storms that resuspend dust from the valley floor. For many decades, rotors and smallerscale vortices generated in the lee of mountains have been known to be hazardous to aircraft. For example, United Airlines Flight 585 crashed near the Colorado Springs Airport in 1991, and the National Transportation Safety Board (NTSB) judged rotors to be a possible contributing factor (National Transportation Safety Board 2001). However, an insufficient understanding of the encountered turbulence prevented direct attribution of the causes for the accident.

There have been several previous studies dedicated to the complex flows in the lee of mountains. During the early 1950s, the Sierra Wave Project and the Jet Stream Project used sailplane measurements with ground tracking to categorize characteristic modes of mountain-wave and rotor phenomena (see, e.g., Holmboe and Klieforth 1957; Grubišić and Lewis 2004). Lidar radial velocity data acquired near Boulder, Colorado, in February of 1987 allowed Ralph et al. (1997) to calculate streamlines and velocity vectors that exhibit full-scale rotor behavior. An important difference between their approach and that presented below was that they utilized the twodimensional continuity equation, whereas in this work a second Doppler lidar supplied the additional information required to extract velocity vectors.

The Mountain-Induced Clear Air Turbulence Experiment (MCAT) was commissioned through a collaboration by the Federal Aviation Administration (FAA), the National Center for Atmospheric Research (NCAR), and the National Oceanic and Atmospheric Administration (NOAA) to address the effects of strong winds 
on aircraft in the area near Colorado Springs in 1997. Darby and Poulos (2006) compared lidar measurements from MCAT with numerical models (horizontal wind components; theoretical versus measured results) during periods of lee wave and rotor interaction. However, it has been difficult to characterize rotors in sufficient detail to relate descriptions of intense, instantaneous turbulence (encountered by aircraft) with mean results from low-resolution numerical models. As mentioned above (Doyle and Durran 2002, 2007), higher-resolution models can now simulate more detailed fluid dynamics behind mountains, helping to close the gap between nearinstantaneous aircraft responses and results from traditional models that capture mean effects. Likewise, the spatially and temporally resolved measurements described below help to fill this gap in understanding and also serve to validate whether simulated rotor dynamics occur in the atmosphere.

Progress on applications such as air pollution management of alpine valleys and aircraft safety for airports near mountains must be based on a sound theoretical understanding of coupled mountain-wave-boundary layer systems. Wurtele et al. (1993) describe a lee wave as a propagation of a disturbance in a density stratified fluid that occurs because of flow over an obstacle. Both linear and nonlinear theories have been developed to describe lee waves mathematically (Lyra 1943; Queney 1955; Queney et al. 1960; Nicholls 1973; Smith 1988; Kuettner 1959; Smith 1976). A detailed history and recent advances in lee wave-rotor theory-simulations are given in the literature review of lee wave measurement by Grubišić and Lewis (2004) for the Sierra Wave Project, in preparation for the Terrain-Induced Rotor Experiment (T-REX) campaign.

\section{b. Terrain-Induced Rotors Experiment}

The Terrain-Induced Rotor Experiment was conducted during March and April of 2006 in the southern Sierra Nevada mountain range in Owens Valley, California. The principal goal of the experiment was to further current understanding and predictability of the dynamics in the mountain-wave rotor-boundary layer (MWRBL) system (Grubišić et al. 2004). Specific goals for the experiment were to understand (i) the role of the upstream flow properties in determining the dynamics and structure of rotors, (ii) wave-rotor dynamic interactions, (iii) internal rotor structure, (iv) rotor-boundary layer interactions, and (v) upper-level gravity breaking and turbulence.

A wide variety of in situ and remote sensors (both ground-based and airborne) was deployed in support of T-REX. In addition to the coherent Doppler lidars, other ground-based instrumentation included the NCAR Raman-Shifted Eye-safe Aerosol Lidar (REAL), ther- mosondes, sodar/RASS, flux towers, the NCAR Integrated Sounding System Multiple Antenna Profiler Radar, and HOBO weather stations (see Grubišić et al. 2004 for a more exhaustive list). Aircraft-based measurements were collected by the NCAR Gulfstream V [HighPerformance Instrumented Airborne Platform for Environmental Research (HIAPER)], the Wyoming King-Air, and the Facility for Airborne Atmospheric Research (FAAM) BAE 146 aircraft, which is collaboratively deployed through the British Met Office and the Natural Environment Research Council.

Researchers from Arizona State University (ASU) and the Deutsches Zentrum für Luft- und Raumfahrt (DLR) coordinated their coherent Doppler lidars to scan a given RHI plane in the hope that two-dimensional radial velocity measurements could be retrieved. ASU had previously collaborated with another lidar group during the Joint Urban 2003 Experiment (JU2003) to coscan a plan position indicator (PPI) plane and to retrieve vectors in the PPI plane (see Newsom et al. 2008). ASU and DLR anticipated that this could allow rotors and other smallerscale vortical structures to be identified and characterized on a cross-barrier plane, providing results directly pertaining to the scientific objectives of T-REX.

\section{c. T-REX operational setup}

The T-REX experiment consisted of two types of coordinated measurement periods. Five enhanced observational periods (EOPs) began at 1500 Pacific standard time (PST) and ended ( $21 \mathrm{~h}$ later) at 1200 (noon) the next day. The durations of the 15 intensive observing periods (IOPs) ranged from only $4 \mathrm{~h}$ to more than a day and a half. IOPs 6 and 13 (for 24-26 March and 14-16 April 2006, respectively) were examples of time windows when both lidars primarily performed coplanar, cross-barrier range-height indicator scans. During other periods, lidar measurement schemes were generally composed of a mix of RHI and plan position indicator scans designed to capture various other features of the flow field.

This paper utilizes data from IOP 6 on 25 March 2006. Our purpose was to capture rotor and subrotor activity with direct measurements (i.e., not requiring complex data assimilation approaches or constraining assumptions). The direct measurements can be used to test both traditional numerical models and retrievals incorporating a mix of simulation and measurement technologies. The Doppler lidars were similar to $2-\mu \mathrm{m}$ WindTracer instruments, built by Lockheed Martin Coherent Technologies, Inc., in Lafayette, Colorado, with $500-\mathrm{Hz}$ pulse repetition frequencies (see Frehlich et al. 1994). They were positioned approximately $2.9 \mathrm{~km}$ apart with the DLR lidar west-southwest of the ASU lidar, along the 
$80^{\circ}$ azimuth of the cross valley transect as shown in Fig. 1. The mean range gate lengths for the DLR and ASU lidars were set to approximately 105 and $87 \mathrm{~m}$, respectively. The global coordinates of the ASU lidar were $36.7975^{\circ} \mathrm{N}$ and $118.1758^{\circ} \mathrm{W}$, and those of the DLR lidar were $36.7926^{\circ} \mathrm{N}$ and $118.2092^{\circ} \mathrm{W}$. The altitudes of the ASU and DLR lidars were 1179 and $1240 \mathrm{~m}$ MSL, respectively. Spatial positioning and temporal synchrony between lidars were crosschecked by comparing their signal-to-noise ratio (SNR) returns from the passage of unique cloud patterns.

\section{d. Dual-Doppler lidar}

The use of a single coherent Doppler lidar to study the detailed fluid mechanics of atmospheric flows is well documented (see Banta et al. 1995, 1996, 1997; Xia et al. 2008). Various forms of dual-Doppler arrangements have been utilized, differing essentially in the type of spatialtemporal overlap and the degree of coordination between lidars. Calhoun et al. (2004, 2006) presented algorithms for wind velocity profiles from coordinated Doppler lidars at a series of intersections upstream of a downtown urban area. The scanning techniques were based on a set of coordinated intersecting vertical (RHI) scans. The extracted vertical profiles of horizontal velocity vectors, or "virtual towers," were placed upwind of urban center in order to assess the effect of increased roughness on the mean flow. Collier et al. 2005 used a configuration that sought to intersect lidar beams at precise points in space. Davies et al. 2005 describe an analysis of errors associated with dual-lidar turbulence measurements. Newsom et al. (2008) were able to retrieve horizontal velocity fields from overlapping PPI scans during JU2003 and noted the tendency for elongation of turbulent structures in the direction of mean flow during less convective morning conditions. The least squares algorithm utilized in this paper is mathematically related to Newsom et al. (2008).

\section{Retrieval algorithm}

\section{a. Space-time window}

The radial velocities from each RHI scan are converted from polar coordinates to Cartesian space by a straightforward conversion to simplify matrix indexing and manipulation. The uniform Cartesian grid spacing is created with the grid cell size dependent on the desired spatial averaging to best suit the data. The finest cells allowable in order to prevent the occurrence of empty grid cells, given our desired temporal resolution ranges, were approximately $130 \times 130 \mathrm{~m}^{2}$.

Once the radial velocities are associated with a particular grid cell, according to the Cartesian coordinate

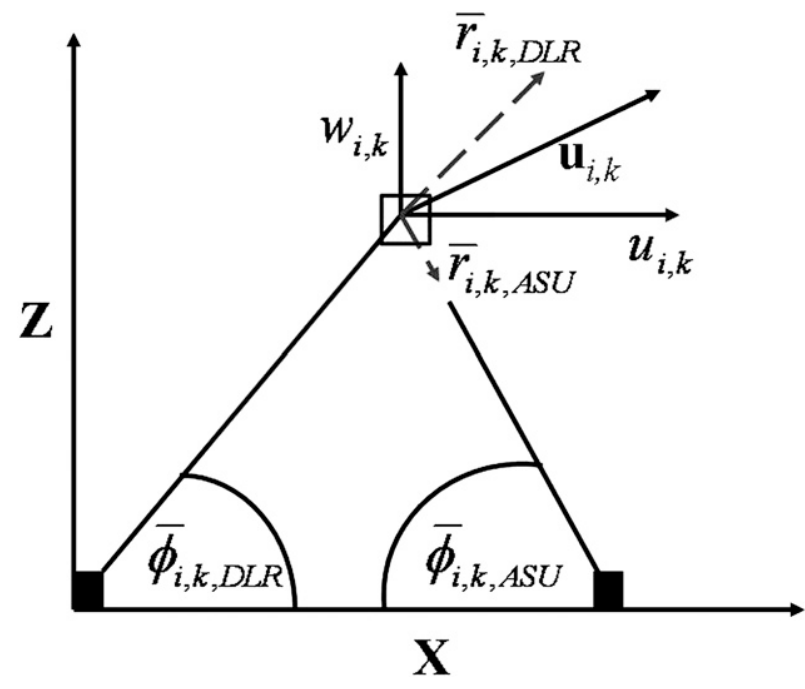

FIG. 3. Schematic of coplane lidar scanning.

transformation, they are summed within each grid cell and over a specified time window, $\Delta t$, (typically 40 to $50 \mathrm{~s}$ ) to determine a local average. Similarly, the mean elevation angle is used for each grid space. Equations (1) and (2) below outline this averaging technique. Note that the superscript indicates the quantity of radial velocities in a particular grid cell during a given time window, $\Delta t$. Schematics of the scanning overlap between instruments and the averaging methods are shown in Figs. 2 and 3, respectively. The variables $r_{i, k}^{n}$ and $\phi_{i, k}^{n}$ represent the radial velocity and elevation angle instance $n$ for grid space $i, k$; variables $\bar{r}_{i, k}$ and $\bar{\phi}_{i, k}$ are the mean radial velocity and mean elevation angles during a given time interval. And $N$ denotes total instances of radial velocity and angles $n$ during the given time interval in grid space $i, k$.

$$
\begin{aligned}
& \bar{r}_{i, k}=\frac{\sum_{n=1}^{N} r_{i, k}^{n}}{N}, \text { where } r_{i, k}^{n}=r_{n}\left(x_{i}, z_{k}\right) \text { for } \\
& \quad \text { grid space, } i, k,
\end{aligned}
$$

$$
\bar{\phi}_{i, k}=\frac{\sum_{n=1}^{N} \phi_{i, k}^{n}}{N}, \text { where } \phi_{i, k}^{n}=\phi_{n}\left(x_{i}, z_{k}\right) .
$$

Consider two radial velocity products (with their associated ranges and elevation angles), one from each lidar, which happen to fall within a given space-time window. Utilizing the geometrical relationship between vectors and radial velocities, a simple system of two equations can be written as in Eqs. (3) and (4), where $u_{i, k}$ and $w_{i, k}$ are the horizontal and vertical wind components and $i, k$ are indices designating position on the Cartesian grid. To determine $u_{i, k}$ and $w_{i, k}$, a least squares 
(a)

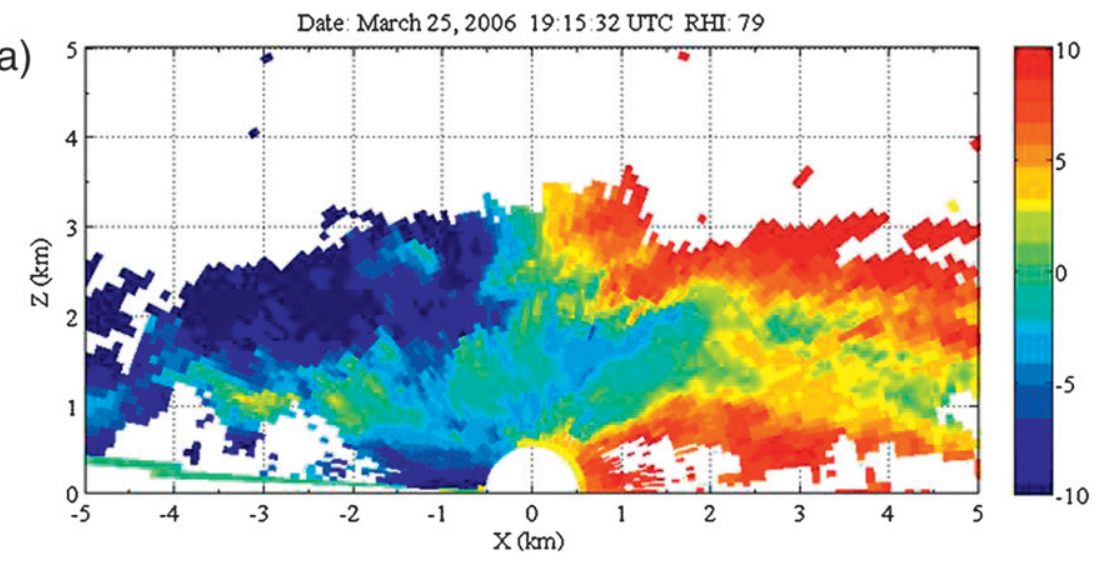

(b)

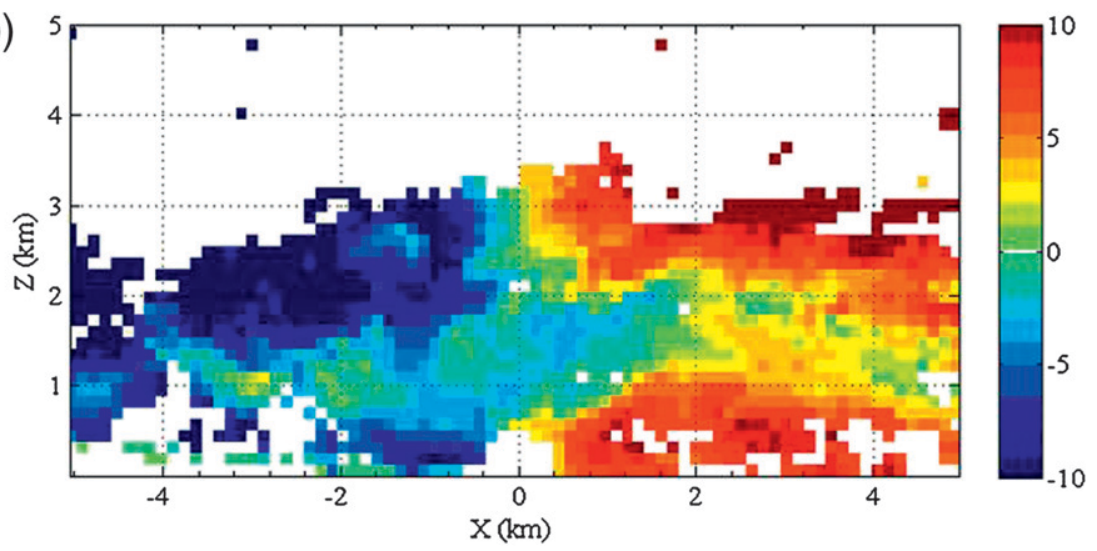

FIG. 4. RHI scan, 1115 PST (1915 UTC) 25 Mar 2006 ASU lidar azimuth $79^{\circ}$. Radial velocities $\left(\mathrm{m} \mathrm{s}^{-1}\right)$ with SNR $>-8$ are shown in (a) standard spherical coordinates and (b) the conversion to Cartesian grid, $130 \mathrm{~m} \times 130 \mathrm{~m}$. Lidar is positioned at origin.

regression is used after Newsom et al. (2008). Figure 4 shows a typical radial velocity RHI scan for the ASU lidar and an example of the corresponding transformation into Cartesian space. Note that the data above $3 \mathrm{~km}$ are principally noise and have been removed.

$$
\begin{aligned}
& \bar{r}_{i, k, \mathrm{ASU}}=u_{i, k} \cos \bar{\phi}_{i, k, \mathrm{ASU}}+w_{i, k} \sin \bar{\phi}_{i, k, \mathrm{ASU}}, \\
& \bar{r}_{i, k, \mathrm{DLR}}=u_{i, k} \cos \bar{\phi}_{i, k, \mathrm{DLR}}+w_{i, k} \sin \bar{\phi}_{i, k, \mathrm{DLR}} .
\end{aligned}
$$

\section{b. Least squares algorithm}

To simplify the notation, the solution is considered for each individual grid cell, where the indices $i$ and $k$ appear in the expressions for both lidars. Equations (3) and (4) can be written more simply as Eq. (5), with the identify of each lidar distinguished with the index $p$, where $p=$ 1,2 . Bin averaging in the notation is also implied in this section. The model equation, or predicted response $f\left(\phi_{p}\right)$, is defined by the regressor variables of vector $\mathbf{g}_{p}=\mathbf{g}\left(\phi_{p}\right)$, which are the cosine and sine of a given elevation angle, $\phi_{p}$, multiplied by the unknown coefficients. These coefficients, $\mathbf{u}$, are estimates of the horizontal and vertical velocities, $u$ and $w$, shown as a dot product of vectors in Eq. (6):

$$
f\left(\phi_{p}\right)=u \cos \phi_{p}+w \sin \phi_{p}
$$

$v$ is perpendicular to the cross-barrier plane,

$$
f\left(\phi_{p}\right)=\mathbf{u} \cdot \mathbf{g}_{p} .
$$

The predicted response and the actual response, the radial velocity $r_{p}$, are equated to create a cost function $S$ from Eq. (7) (Wolberg 2006, 44-48):

$$
S=\sum_{p=1}^{m}\left[r_{p}-f\left(\phi_{p}\right)\right]^{2} .
$$

This cost function is minimized with respect to each coefficient $\partial S / \partial a_{q}=0$, where $q=1,2$, and $a_{1}=u$, and $a_{2}=w$. This leads to Eq. (8):

$$
\sum_{p=1}^{m} f\left(\phi_{p}\right) \frac{\partial f\left(\phi_{p}\right)}{\partial a_{q}}=\sum_{p=1}^{m} r_{p} \frac{\partial f\left(\phi_{p}\right)}{\partial a_{q}} .
$$


The individual regressor variables from Eqs. (9) and (10) are inserted into the above equation:

$$
\begin{gathered}
g_{p, 1}=\frac{\partial f\left(\phi_{p}\right)}{\partial a_{1}}=\cos \phi_{p}, \\
g_{p, 2}=\frac{\partial f\left(\phi_{p}\right)}{\partial a_{2}}=\sin \phi_{p} .
\end{gathered}
$$

Equation (11) enumerates the full $\mathbf{g}$ matrix. This leads to the least squares normal equations in matrix form in Eq. (12) (Montgomery et al. 2006):

$$
\begin{aligned}
\mathbf{g} & =\left[\begin{array}{ll}
\cos \phi_{1} & \sin \phi_{1} \\
\cos \phi_{2} & \sin \phi_{2}
\end{array}\right], \\
\mathbf{g}^{\prime} \mathbf{g} \mathbf{u} & =\mathbf{g}^{\prime} \mathbf{r} .
\end{aligned}
$$

The expanded matrix $\mathbf{g}^{\prime} \mathbf{g}$ is shown in Eq. (13):

$$
\left.\begin{array}{l}
\cos \phi_{1} \sin \phi_{1}+\cos \phi_{2} \sin \phi_{2} \\
\sin ^{2} \phi_{1}+\sin ^{2} \phi_{2}
\end{array}\right] .
$$

The resultant matrix can now be inverted and multiplied by both sides of Eq. (12) to solve for the horizontal and vertical velocities in each location [Eq. (14)]. To understand the uncertainty behavior within the least squares solution for $u$ and $w$, the inverse of the $\mathbf{g}^{\prime} \mathbf{g}$ matrix is directly calculated. Sine and matrix algebra simplify Eq. (14) below to Eq. (15):

$$
\mathbf{u}=\left(\mathbf{g}^{\prime} \mathbf{g}\right)^{-1} \mathbf{g}^{\prime} \mathbf{r}=\left[\begin{array}{l}
u \\
w
\end{array}\right],
$$

This solution suffers from ill conditioning near the ground as the sines of low elevation angles tend to zero, discussed in more detail below. This causes the determinant to tend to zero, making its reciprocal used in the inverse of the $\mathbf{g}^{\prime} \mathbf{g}$ matrix to become unstable.

A small degree of positional error is well tolerated by the retrieval algorithm because of the space-time averaging window in our retrieval method. This should be compared to approaches expecting precise beam crossing at calculated intersections. Such methods will be relatively less tolerant of pointing and synchrony errors.

\section{c. Quality control}

The SNR fields associated with the radial velocity data for the morning of 25 March show the decay of data quality with increasing radial distance (Fig. 5). SNR, as shown, is measured as the base 10 logarithm of the signal power to noise power ratio. The average SNR for both lidars drops to below $-10 \mathrm{~dB}$ at radial distances beyond $4 \mathrm{~km}$; SNR values below this level in the decibel scale indicate that the ratio of returned signal power to the power of the system noise is less than 0.1 and are consequently considered poor returns. Accordingly, velocity retrievals for this day typically considered data at heights between 0.8 and $2.9 \mathrm{~km}$.

Notice that Fig. 5 shows that there are regions of "better return" (in this case, for the ASU lidar). The usable vertical limit of the ASU lidar appears to be ap- proximately $3.3 \mathrm{~km}$ AGL, since mean SNR is below -10 above this height. Laterally, the acceptable limit is around $4 \mathrm{~km}$ from this lidar. Visual inspection proved an effective first evaluation of the data to recognize levels of noise that are too high for effective processing. Therefore, the spatial window used in subsequent results was selected to provide acceptable levels of noise from both lidars. A low-pass filter for SNR with a minimum cutoff value of $-10 \mathrm{~dB}$ prevented the inclusion of much of the noise. The maximum height for acceptable average SNR is a consequence of the relief of the valley (approximately $3000 \mathrm{~m} \mathrm{AGL).} \mathrm{Flow} \mathrm{above} \mathrm{this} \mathrm{height}$ was on average more likely to contain lower aerosol levels when no clouds were present, compared to airflow passing closer to the side of the mountain.

\section{Vortex identification}

\section{a. Recognition of a vortex}

A rotor is defined as a type of vortex in the mountainwave-boundary layer system with a spanwise horizontal axis typically oriented parallel to the valley or mountain ridgeline (Doyle and Durran 2002). Nonetheless, ambiguities in the accepted definition of a vortex complicate definitions of rotors and subrotors. It would seem straightforward to assume that a region of high vorticity and consequently circulation would indicate the presence 


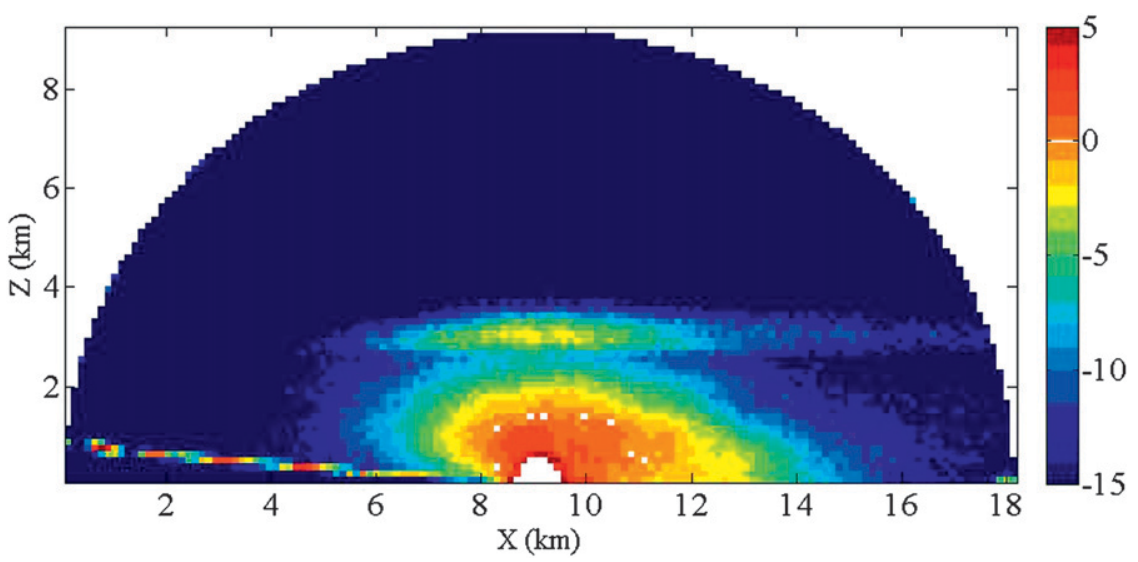

FIG. 5. Time-mean SNR (in dB summed over $100 \mathrm{~m} \times 100 \mathrm{~m}$ grid) for given measurements over two hours from 0904 to 1117 PST (1704 to 1917 UTC) 25 May 2006 for ASU lidar.

of a vortex. However, as can be seen in the literature, there has been a need to deepen the definition of a vortex (see, e.g., Hunt et al. 1988; Lugt (1979); Chong et al. 1990). Jeong and Hussain (1995) describe the implications and caveats of previous identifications and definitions of a vortex core. They suggest two principal requirements for an identified vortex core: (i) it must contain net vorticity and (ii) its geometry should be the Galilean invariant. Limitations exist for the three common indicators: pressure minima, spiraling or closed streamlinespath lines, and constant isovorticity surfaces. The minimum pressure condition and vorticity can have different scales in examples such as the Lamb vortex and Burger's vortex, allowing for pressure and vorticity to become disassociated at various distances from the axis of the core. An unsteady strain rate may cause a pressure minimum or eliminate a minimum altogether in a vortical flow (Calhoun 1998). The closed path line condition fails because particles may not complete a full revolution around the center of a vortex in its lifetime. Finally, regions with high vorticity can be biased by shear and frame of reference while lacking a vortex core. Therefore, Jeong and Hussain (1995) propose a negative $\lambda_{2}$ eigenvalue approach for the identification of a vortex region. They suggest that their scheme prevents the incorrect identification of a region that may have shear and an associated rotation without having a true vortex structure. Chong et al.'s (1990) critical point analysis is also applied in the literature, as done by Chacín and Cantwell (1997) in a direct numerical simulation of flat plate flow that suggests that vortices tend toward either a state of stable vortex stretching or an unstable asymptotic state.

\section{b. Swirling strength}

The present dual coherent lidar analysis considers only the flow field in a two-dimensional plane, allowing sim- plifications in the critical point analysis. Local velocity gradient tensors are readily calculated from individual points in a gridded two-dimensional flow field (Zhou et al. 1999; Adrian et al. 2000). This two-dimensional velocity gradient matrix has two real eigenvalues, $\lambda_{\text {cr }}$ or a pair of complex conjugate eigenvalues $\lambda_{\text {cr }} \pm i \lambda_{\text {ci }}$, as shown in Eqs. (16) and (17). The reciprocal of the complex part of the eigenvalue, $\lambda_{\text {ci }}$, is defined as swirling strength and corresponds to the period required for a particle to rotate around a center axis of the local vortex. However, swirling strength does not specify the orientation of swirl, so it can be coupled with vorticity, $\omega$, to provide a more complete picture. This configuration only provides the swirl relative to the plane of measurement, yet vortex cores evolve in three dimensions. Although the Sierra Nevada mountain range is sometimes idealized for theoretical or model purposes as uniform in the direction transverse to the cross-barrier flow, clearly, there will be three-dimensional effects that occur in the flow, and this must be kept in mind when viewing the results below:

$$
\begin{aligned}
& \mathbf{D}^{2 \mathrm{D}}=\left[\begin{array}{ll}
\frac{\partial u_{1}}{\partial x_{1}} & \frac{\partial u_{1}}{\partial x_{2}} \\
\frac{\partial u_{2}}{\partial x_{1}} & \frac{\partial u_{2}}{\partial x_{2}}
\end{array}\right], \\
& \left|\begin{array}{ll}
\frac{\partial u_{1}}{\partial x_{1}}-\lambda & \frac{\partial u_{1}}{\partial x_{2}} \\
\frac{\partial u_{2}}{\partial x_{1}} & \frac{\partial u_{2}}{\partial x_{2}}-\lambda
\end{array}\right|=0,
\end{aligned}
$$

with eigenvalues $\lambda_{\mathrm{cr}, 1}, \lambda_{\mathrm{cr}, 2}$ or $\quad \lambda_{\mathrm{cr}} \pm i \lambda_{\mathrm{ci}}$.

The local velocity gradient tensor, $\mathbf{D}^{2 \mathrm{D}}$, used to calculate the local swirling strength is determined for each grid cell by centered second-order finite differences. The 
(a)

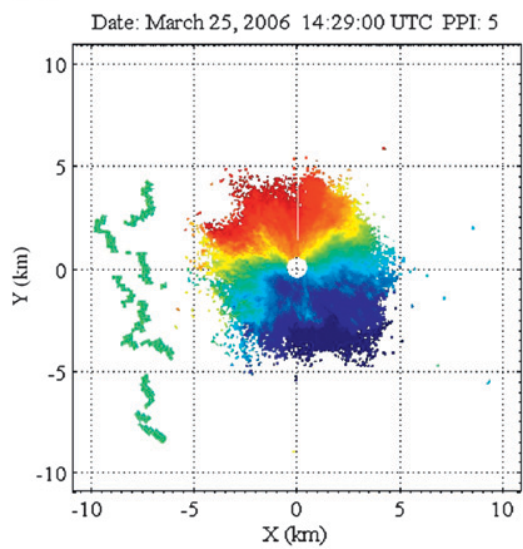

(b)

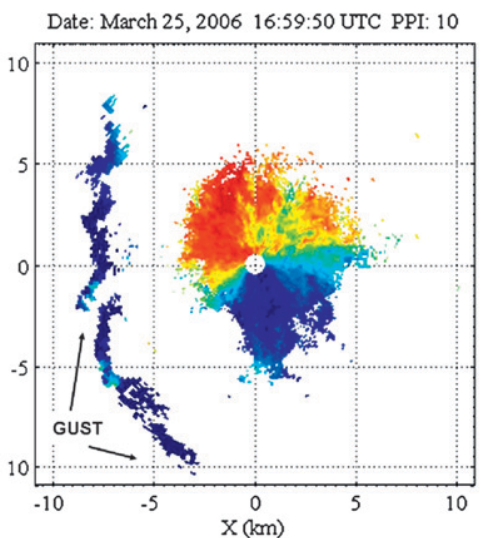

(c)

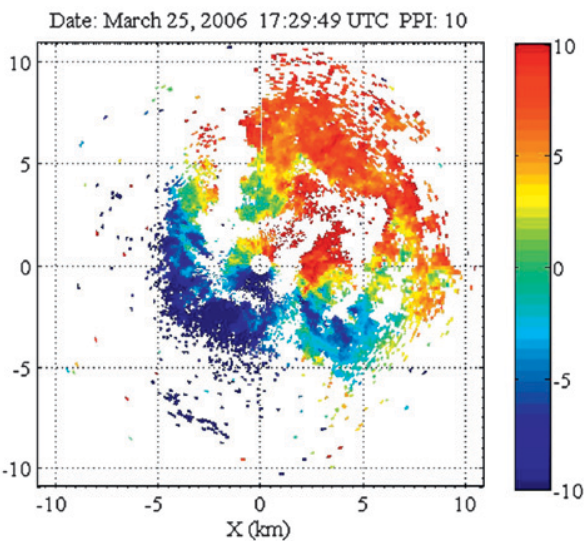

FIG. 6. DLR PPI scans of radial velocity $\left(\mathrm{m} \mathrm{s}^{-1}\right.$ ). Elevation angle (a) 5 $5^{\circ}$ at 0629:00 PST (1429:00 UTC) 25 Mar 2006; flow is from the south at approximately $165^{\circ}$ with a maximum magnitude of 11 to $12 \mathrm{~m} \mathrm{~s}^{-1}$. (b) Elevation angle 10 at 0859:50 PST (1659:50 UTC); flow is from the south at $170^{\circ}$ with a maximum velocity of $10 \mathrm{~m} \mathrm{~s}^{-1}$; note the incoming pulse of the wind from the west over the mountains. (c) Elevation angle $10^{\circ}$ at 0929:49 PST (1729:49 UTC); flow is from the southwest at $220^{\circ}$ with a maximum velocity of $13 \mathrm{~m} \mathrm{~s}{ }^{-1}$; the mean flow has become cross-valley, and the lower up-valley mean flow has been destroyed.

eigenvalues that determine the local swirling strength are derived from this tensor. Streamlines are determined by a cubic MATLAB function streamslice. Results from this analysis are depicted in combinations of velocity fields, vorticity, swirling strength, and streamlines to show levels of correlation. The shading interpolation scheme used to produce smoothed vorticity contours from the interp MATLAB function is based on Fritsch and Carlson's (1980) cubic interpolation algorithm for monotone data to improve visual appeal.

\section{Meteorological overview-25 March 2006}

The regional meteorology of Owens Valley on 25 March 2006 was characterized by an incoming low pressure system (trough) moving eastward from beyond the California coastline. The high to low pressure gradient induced a pressure-driven flow, which moved from south to north. At 0530 PST (1330 UTC), the maximum flow speed was $5 \mathrm{~m} \mathrm{~s}^{-1}$ upvalley (from southeast to northwest). Since this time was prior to sunrise, the flow direction was counter to the anticipated katabatic, downvalley motion. From the ground level to approximately $700 \mathrm{~m}$ AGL, there was a slight counterclockwise (positive) twisting of the flow vectors and then a clockwise return at higher altitudes for the next hour. A conical PPI scan at an elevation angle of $5^{\circ}$ is seen in Fig. 6 a for a minute in this time period.

Later, at 0700 PST (1500 UTC), the orientation of the lower-level winds maintained the south-southeast to north-northwest direction, but the magnitude of the flow velocity increased slightly to approximately $10 \mathrm{~m} \mathrm{~s}^{-1}$, and strong $\left(>15 \mathrm{~m} \mathrm{~s}^{-1}\right)$ westerly gusts began to intermittently penetrate deeper into the valley boundary layer. Westerly pulses of momentum lasted up to 20 and $30 \mathrm{~min}$. These gusts traveled down the lee side of the mountain, severely disrupting the consistent up-valley flow that had been established by the pressure gradient (trough) and was strengthened by the radiative surface heating.

Figure $6 \mathrm{~b}$ shows a strong gust descending over the mountaintop, which broke up the mean up-valley flow. The ability of the lidar to capture the incoming elevated gust of westward winds (see dark region of lidar signal to the left of Fig. 6b) was likely associated with enhanced backscatter return associated with low-level clouds rolling over the mountain range (or, perhaps less likely, with the lofting and suspension of snow or dust caused by the impingement of strong cross-barrier winds on the surface upwind). The up-valley flow was shown to be fully displaced 30 min later by this gust and the mean flow has become cross-valley, as seen in Fig. 6c. It is likely that the penetration of the high-momentum air into the valley was not regular along the valley because of the peaks, passes, and gaps in the Sierra Nevada; note that these results show a two-dimensional window of a complex threedimensional flow.

This pattern continued-that is, the up-valley flow reformed gradually with the subsidence of each eastward pulse of momentum, to be disturbed later by increasingly strong winds up to $25 \mathrm{~m} \mathrm{~s}^{-1}$ by 1030 PST (1830 UTC). By 1330 PST (1930 UTC), violent mixing at heights below $3 \mathrm{~km}$ was accompanied by brief but extensive cloud cover and light precipitation at 1530 PST (2130 UTC). In the late afternoon (1800 PST), low 
(a)

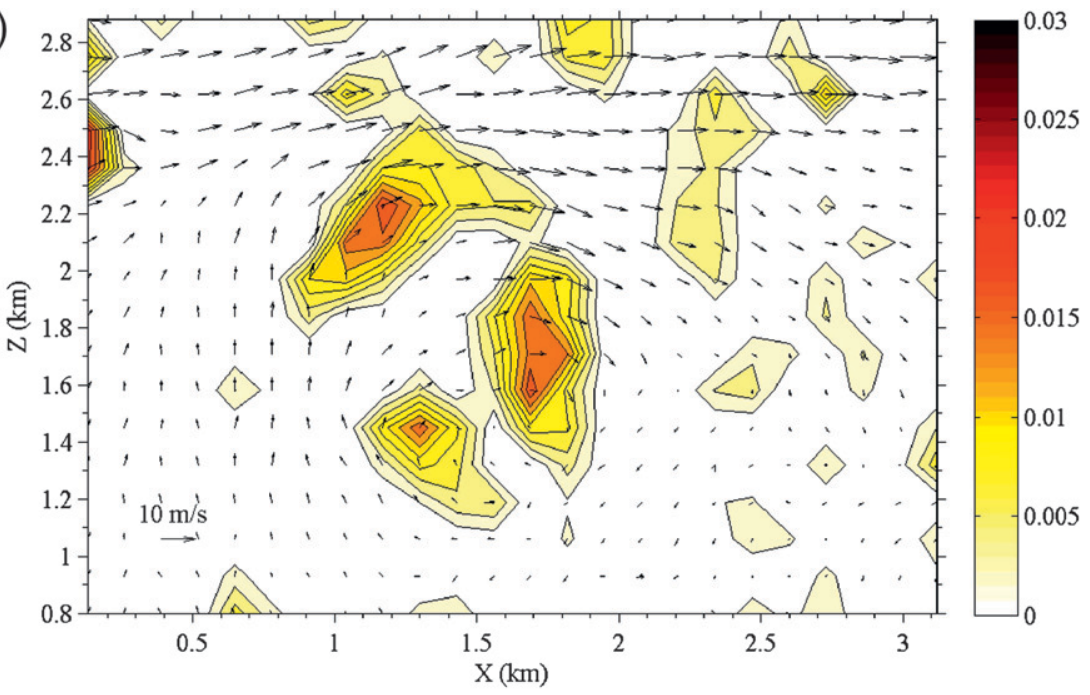

(b)

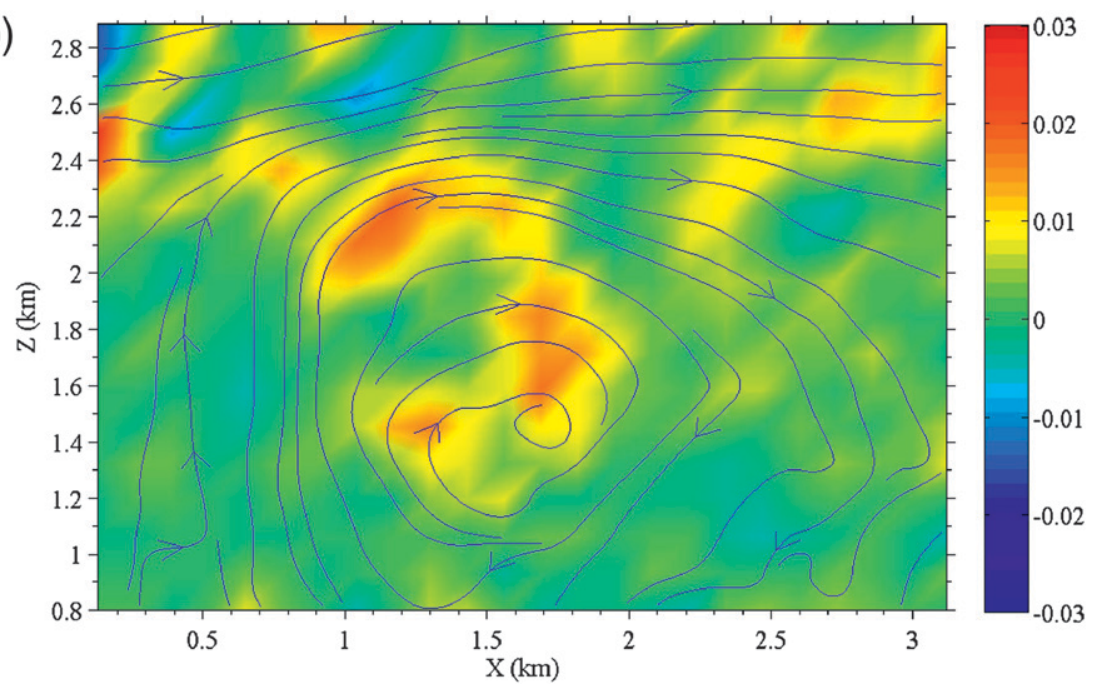

FIG. 7. Rotor at 1104 PST (1904 UTC): (a) 2D velocity field and swirling strength contours; (b) streamlines (with arrows) and vorticity. Units of vorticity and swirling strength are $\mathrm{s}^{-1}$.

aerosol content created poor sensing conditions for the coherent Doppler lidars.

\section{Results}

\section{a. Rotors}

Two-dimensional velocity vectors retrieved from the coscanned, cross-barrier plane provide a wealth of information on the existence, nature, and behavior of vortical flows below the level of the rotor clouds. In particular, data from IOP 6 from 0904 to 1117 PST (1704-1917 UTC) 25 March 2006 shows that rotors and subrotors (in our terminology described below) can be detected in clear air and tracked using the dual-lidar coplanar scanning and retrieval methods given in this paper. During our period of interest, roll clouds were present above our domain, and although they were almost coherently aligned parallel to the mountain ridge, they showed a three-dimensional variability presumably due to their updrafts and downdrafts. Therefore, vorticity not visible on our plane of visualization may also be significant.

For the purposes of discussion in this paper, rotors and subrotors are given the following distinctions: A rotor will be classified as an event with a region of coherently curving streamlines around a center point spanning a diameter of at least $1 \mathrm{~km}$. The coherency of a rotor is sustained for at least multiple minutes; rather than advecting with the outer flow downstream, it is associated with and likely sustained by the juxtaposition of a higher-level eastward-moving flow with a lower-level reverse flow. However, the time mean swirling strength is less dense 
(a)

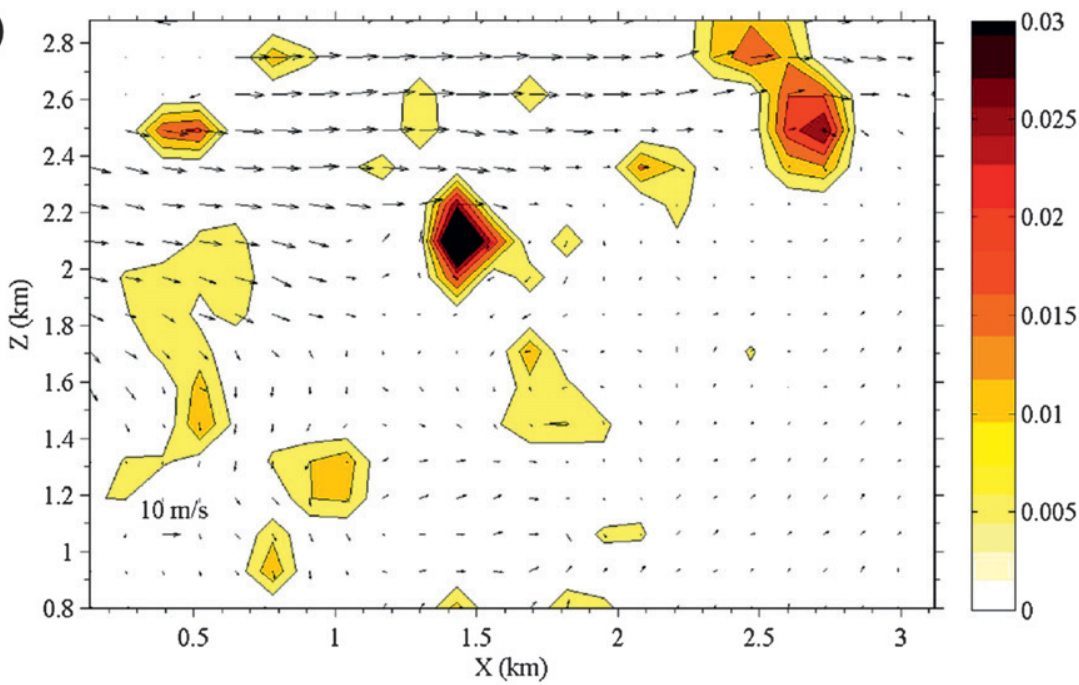

(b)

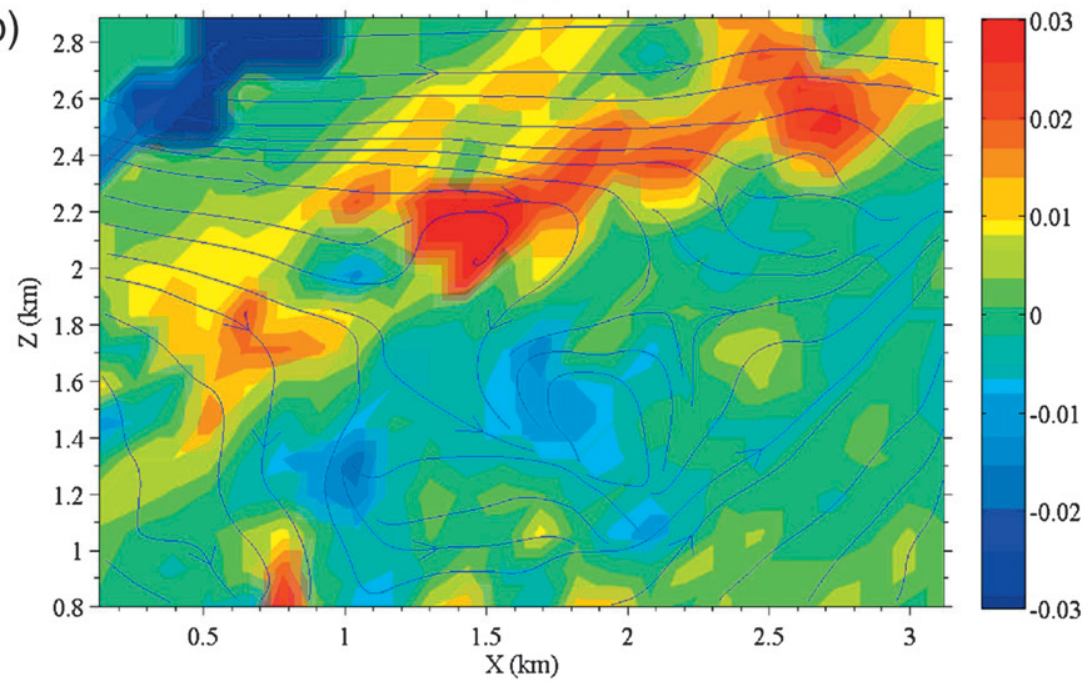

FIG. 8. (a) 2D velocity field and swirling strength contours and (b) streamlines (with arrows) and vorticity for subrotors at 1109 PST (1909 UTC). Units of vorticity and swirling strength are $\mathrm{s}^{-1}$.

than subrotors and the behavior is less coherent, in agreement with 3D results produced by Doyle and Durran (2007). Therefore, a rotor by our definition is a larger, less coherent, more positionally stable vortical region in the MWRBL. For contrast (see next section), a subrotor is a transient structure (at least on our visualization plane) with a net vorticity and swirling strength greater than $0.01 \mathrm{~s}^{-1}$; it is not necessarily contained within the circulation of the main rotor, but rather more typically advects with the local mean flow (usually eastward).

During our observations, rotors seem often to be generated from shear between opposing flows, for example, when a strong westerly flow meets an opposing undercurrent at heights from 1.2 to $2.2 \mathrm{~km}$. The angle of the incoming higher-altitude flow may allow for a more ideal or larger and complete circulation to form, comprised of a single or multiple vortices. Opposing lower-level flows with sufficient upward velocity may "pinch off" the shear, allowing the formation of a larger independent circulation. These flow regimes typically generated rotors with positive circulation and vorticity (clockwise) in the measurement domain. The local maximum intensity of smaller vortices within the rotor could fluctuate, as could their locations, but the vortices contained inside the larger circulation rarely exceeded $0.02 \mathrm{~s}^{-1}$.

It is typical for the mean horizontal velocity for the entire measurement window (i.e., spatial average $\langle\mathbf{u}\rangle$ over the measurement window during a given $\Delta t$ ) to be $<1.5 \mathrm{~m} \mathrm{~s}^{-1}$ during rotor genesis and only grow to $4.5 \mathrm{~m} \mathrm{~s}^{-1}$ in any direction during the rotor's lifetime. Not surprisingly, recirculations are associated with lower average flowthrough velocities. Following the genesis stage, the rotor may encounter larger-scale flow dynamics. It may be deformed, shifted, or washed out by 

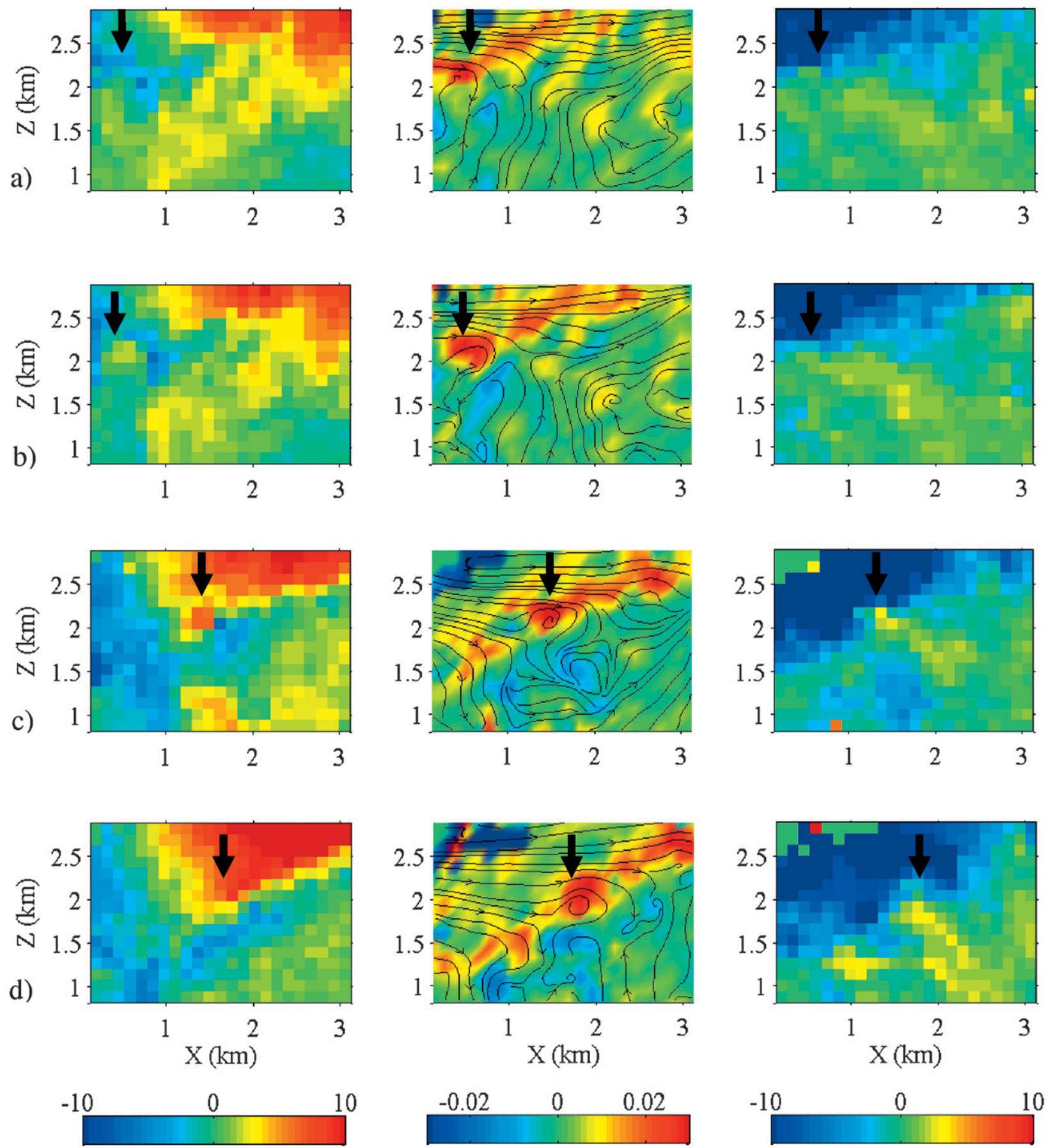

FIG. 9. The evolution of subrotors is shown at (a) 1106, (b) 1107, (c) 1109, and (d) 1110 PST (1906-1910 UTC). A particular subrotor of interest is denoted by arrows. (left) Series of DLR radial velocity graphs $\left(\mathrm{m} \mathrm{s}^{-1}\right)$. (middle) Retrieved results (using both lidars); background color is vorticity $\left(\mathrm{s}^{-1}\right)$ and streamlines (with arrows). (right) ASU radial velocity graphs $\left(\mathrm{m} \mathrm{s}^{-1}\right)$. Each frame has $\Delta t=40 \mathrm{~s}$ bin averaging with a $130 \mathrm{~m} \times 130 \mathrm{~m}$ grid. Note the presence of vorticity that correlates with areas of high-velocity gradients (i.e., abrupt color change) in common area of both graphs.

larger-scale air movements. Figure 7 displays a view of a rotor from 1104 PST (1904 UTC), with velocity vectors and streamlines encircling a core composed of a set of vortices.

\section{b. Subrotors}

As mentioned above, subrotors are defined as relatively concentrated regions of swirling strength that 
(a)

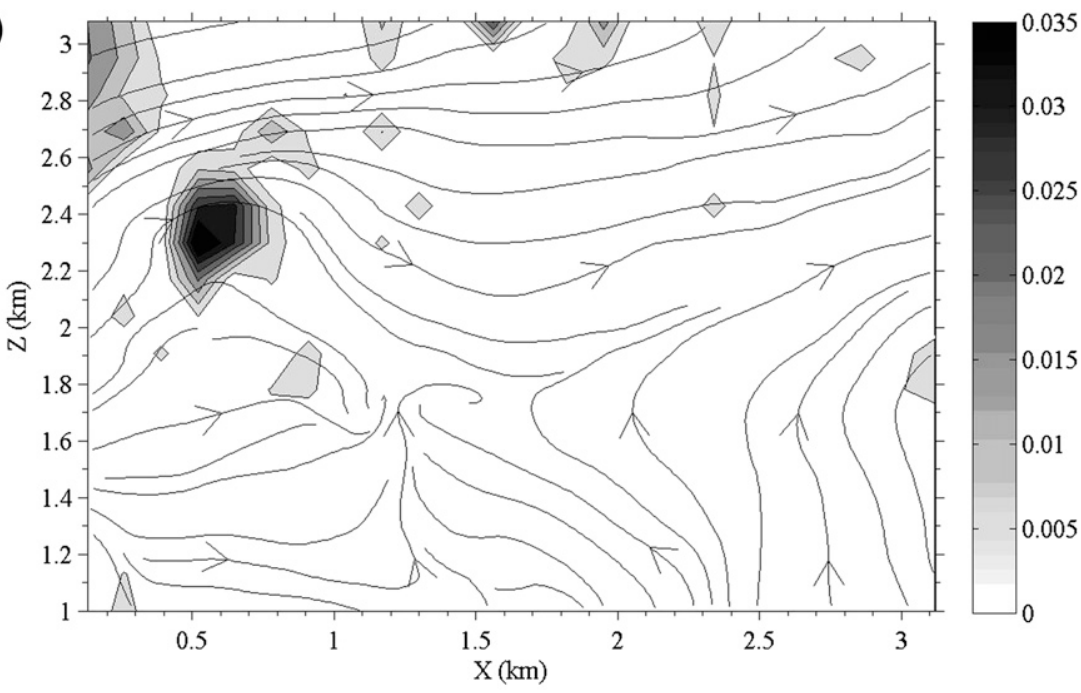

(b)



FIG. 10. Subplots of streamlines (with arrows) and swirling strength (contours) showing rotor advection ( $\mathrm{s}^{-1}$ ): (a) 1054 (1804 UTC), (b) 1009 (1809 UTC), (c) 1011 (1811 UTC), and (d) 1013 PST (1813 UTC). Removed data with SNR $<-10$.

most frequently advect eastward in the mean flow. They do not appear to be necessarily spatially tied to a junction of upper eastward-moving flow and an opposing lower flow. They are seen to both advect in the visualization plane and commonly disappear and reappear, presumably because they have limited spanwise extents and move in and out of the visualization plane. Secondly, these vortices occur on a smaller scale, typically smaller than $1 \mathrm{~km}$ in diameter, with a concentration of vorticity and swirl. Two strong subrotors with positive vorticity and another with opposite orientation are seen in Fig. 8. The concentrated vortex definition agrees well with recent 3D simulations of Doyle and Durran (2007) that observe individual intense vortices that are "swept downstream past the main rotor along the in- terface between the main rotor and the surrounding lee wave."

These subrotors are typically tighter and stronger concentrations of vorticity and swirling strength, which can reach strengths above $0.03 \mathrm{~s}^{-1}$. Vortices of this type can have both positive and negative orientations (clockwise and counterclockwise). These vortices may form in pairs, with apparent interactions between one another. It is typical for isolated subrotors above $2.6 \mathrm{~km}$ AGL to rotate clockwise, or with positive vorticity due to dominant vertical gradients resulting from the eastward horizontal velocity. However, counterclockwise vortices (negative vorticity) often form below $2 \mathrm{~km}$.

At 1106 PST (1906 UTC), flow conditions cause the formation of a subrotor in the two-dimensional wind 
(c)

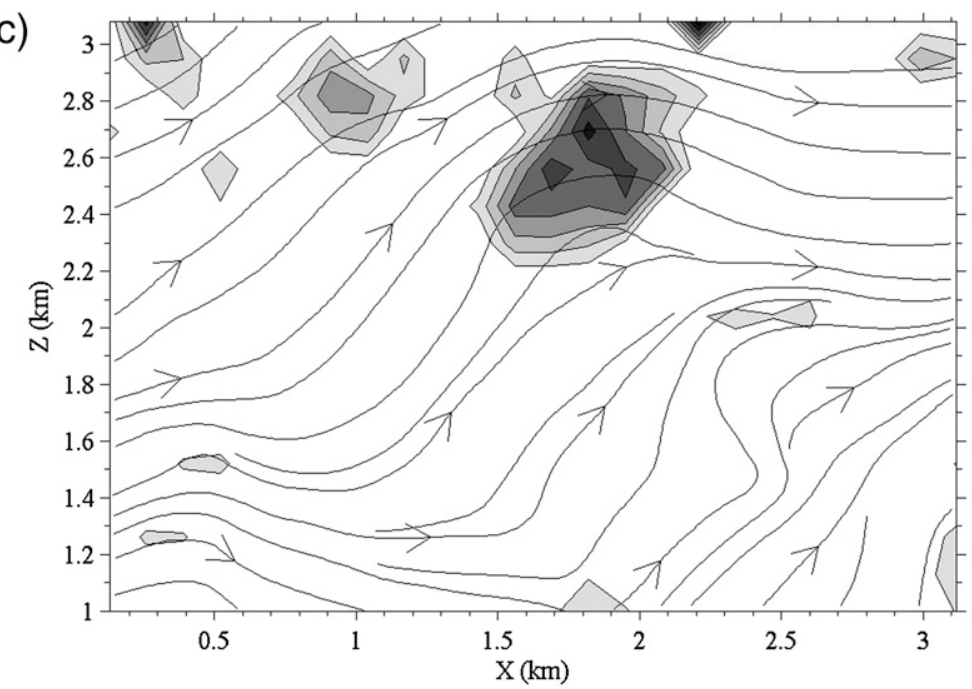

(d)

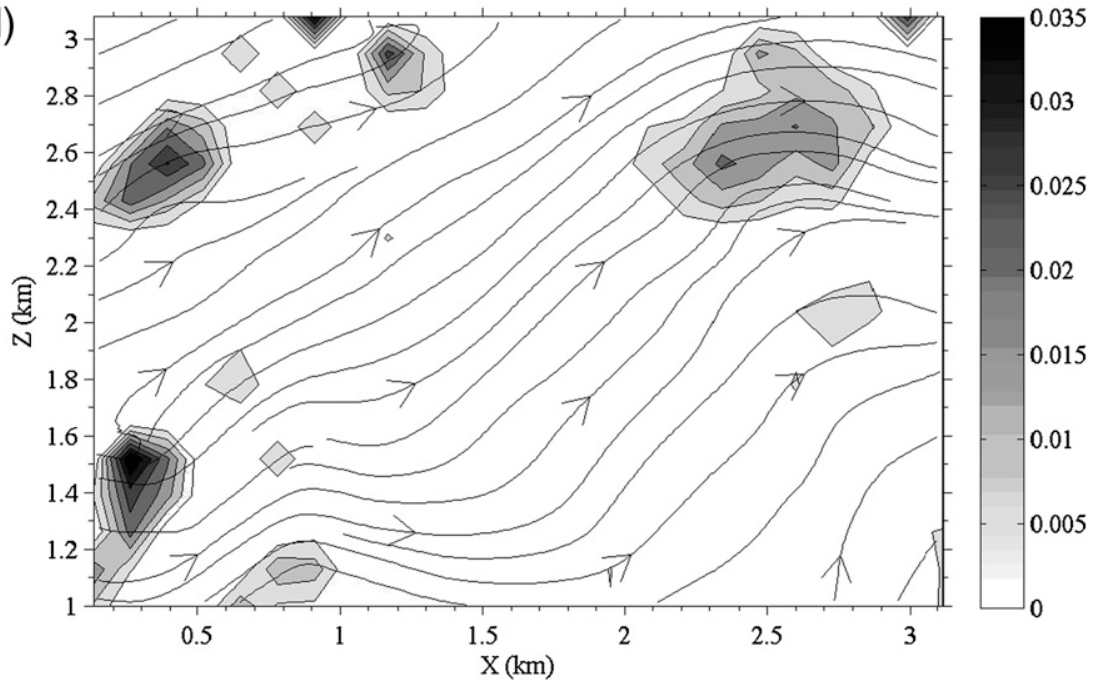

FIG. 10. (Continued)

field (Fig. 9a). It becomes detectable $0.5 \mathrm{~km}$ east of the DLR lidar (approximately $2 \mathrm{~km}$ in height) and advects through the measurement domain (Figs. 9b-d). A substantial current of wind to the east and a brief updraft (1-2 $\mathrm{min}$ ) at $1.5 \mathrm{~km}$ AGL appear to be associated with the formation of the subrotor on the current's edge. It is sustained and intensified as the dominant current pushes it forward while encountering a positive velocity gradient $\partial u / \partial z$ from the opposing lower elevation flow, as discussed previously.

\section{c. Comments on rotors-subrotors classification}

Clearly, our classification of rotors and subrotors has an arbitrary element, especially size or strength criteria. However, it is instructive to contrast the various sizes of vortical motions in order to more clearly ascertain what one means by "rotor." Our concept of a large-scale rotor, and in fact, the conclusions naturally drawn through observations of stable rotor clouds, may belie the level of instantaneous chaos existing below. The mean effect aloft may yield a stable rotor cloud, and the timeor ensemble-averaged streamlines below may indicate a large-scale rotor, even though large-scale rotors may be undetectable instantaneously. Therefore, we expect size determinations of rotors to be a function of the level of averaging. Nevertheless, lidar results do show some evidence of large-scale rotary motions, at least at the level of averaging required by the given retrieval method. However, subrotors unambiguously populate these flows in our time and space window. Additionally, we recognize that this analysis does not rule out the possibility that these smaller-scale vortices, referred to as subrotors in this paper, result from hydraulic jumps at times during the evolution of the flow. For aircraft passing through our 

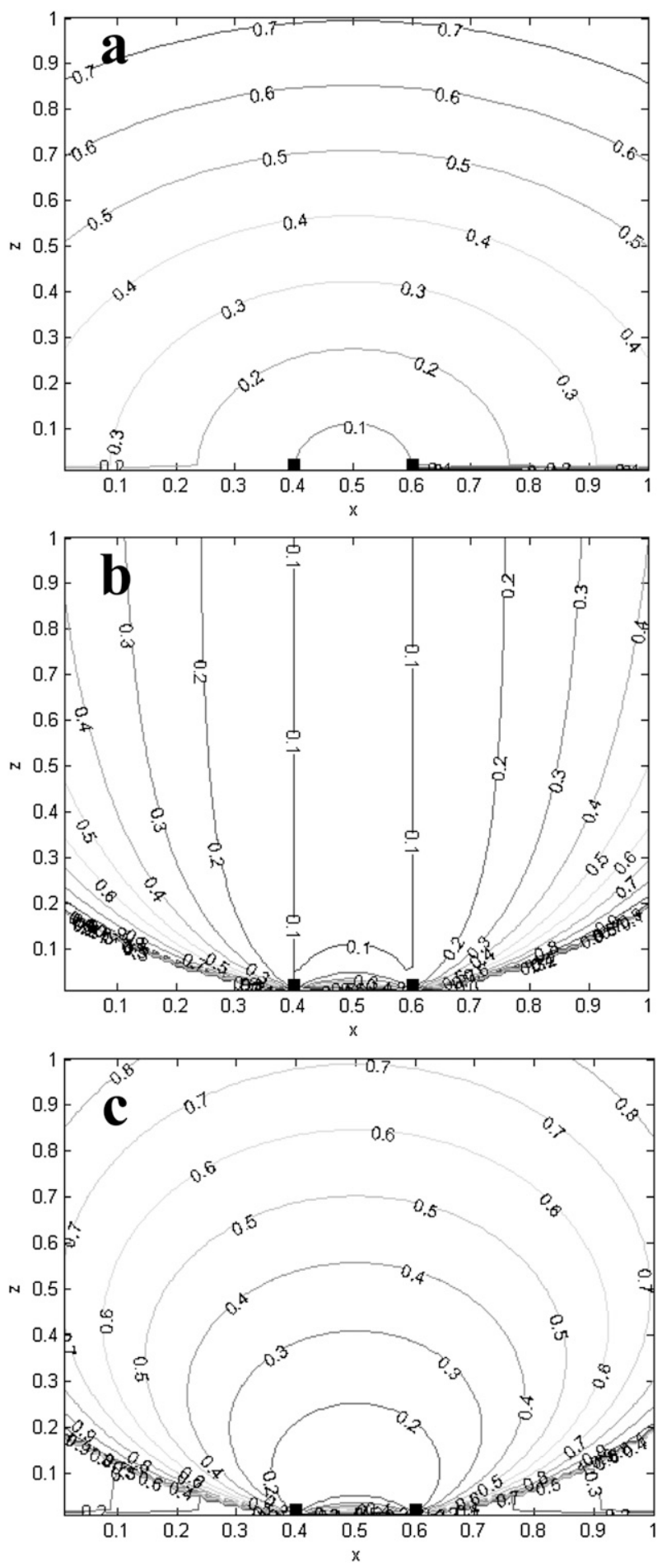

FIG. 11. Direct computation of variances of the least squares solution with respect to position, with the nominal radial uncertainty of 0.1: (a) Uncertainty of $u$ component, (b) uncertainty of $w$ component, (c) geometric uncertainty of $\mathbf{u}$ vector. Lidars are represented at $x$ positions of 0.4 and 0.6 . plane of measurement, the presence of concentrated, powerful subrotors has immediate relevance.

As an example of the difficulty of clearly classifying rotors versus subrotors, see Fig. 10, which shows the 1004 PST (1804 UTC) vortex as it is pushed out of the visualization plane at 1013 PST (1813 UTC) by a strong pulse of cross-barrier momentum. This may be associated either with variations in the position of a lee wave or with fluctuations in downward currents, as the trough of a lee wave (or current) is seen to travel through the plane downstream of the circulation of the vortex.

\section{d. Uncertainty scheme}

An idealized uncertainty scheme was constructed to understand the behavior of the least squares fit of the data. A nondimensional domain was established with uniform longitudinal and azimuthal uncertainty estimates of the velocity measurements, $\varepsilon_{r}$, for each lidar, or $\varepsilon_{r_{1}}$ and $\varepsilon_{r_{2}}$. Actual velocity measurement uncertainty will increase with range. The uncertainty in $u$ and $w$ are determined statistically as shown in Eq. (18) from the least squares retrieval of the of the $\mathbf{u}$ vector, expanded for each velocity component [shown individually in Eqs. (19) and (20)], where $C=\cos \phi_{2} \sin \phi_{1}-\cos \phi_{1} \sin \phi_{2}$.

$$
\begin{aligned}
& \varepsilon_{u_{i}}=\left[\sum_{j=1}^{N}\left(\frac{\partial u_{i}}{\partial r_{j}}\right)^{2}\left(\varepsilon_{r_{j}}\right)^{2}\right]^{1 / 2}, \\
& \varepsilon_{u}=\left[\left(\frac{\sin \phi_{2}}{C}\right)^{2}\left(\varepsilon_{r_{1}}\right)^{2}+\left(\frac{-\sin \phi_{1}}{C}\right)^{2}\left(\varepsilon_{r_{2}}\right)^{2}\right]^{1 / 2}, \\
& \varepsilon_{w}=\left[\left(\frac{-\cos \phi_{2}}{C}\right)^{2}\left(\varepsilon_{r_{1}}\right)^{2}+\left(\frac{\cos \phi_{1}}{C}\right)^{2}\left(\varepsilon_{r_{2}}\right)^{2}\right]^{1 / 2} .
\end{aligned}
$$

The geometric uncertainty, $\varepsilon_{\mathbf{u}}$, can therefore be determined for each coordinate as in Eq. (21); this uncertainty is a function of the determinant of the $\mathbf{g}^{\prime} \mathbf{g}$ matrix and the measurement uncertainty and is comprised of sum of the squares of the horizontal and velocity components. Figures $11 \mathrm{a}$ and $11 \mathrm{~b}$ display the uncertainty associated with the horizontal and vertical velocity components, while Fig. 11c shows the ill conditioning that occurs when $\sin \phi_{1} \approx \sin \phi_{2}$.

$$
\varepsilon_{\mathbf{u}}=\left(\varepsilon_{u}^{2}+\varepsilon_{w}^{2}\right)^{1 / 2}=\frac{\left(\varepsilon_{r_{i}}\right)(2)^{1 / 2}}{|C|}
$$

The contours shown in the previous figures match the behavior given in the analysis of Lhermitte and Miller (1970) for a dual-radar, coscanned plane, as shown in (Fig. 12). 


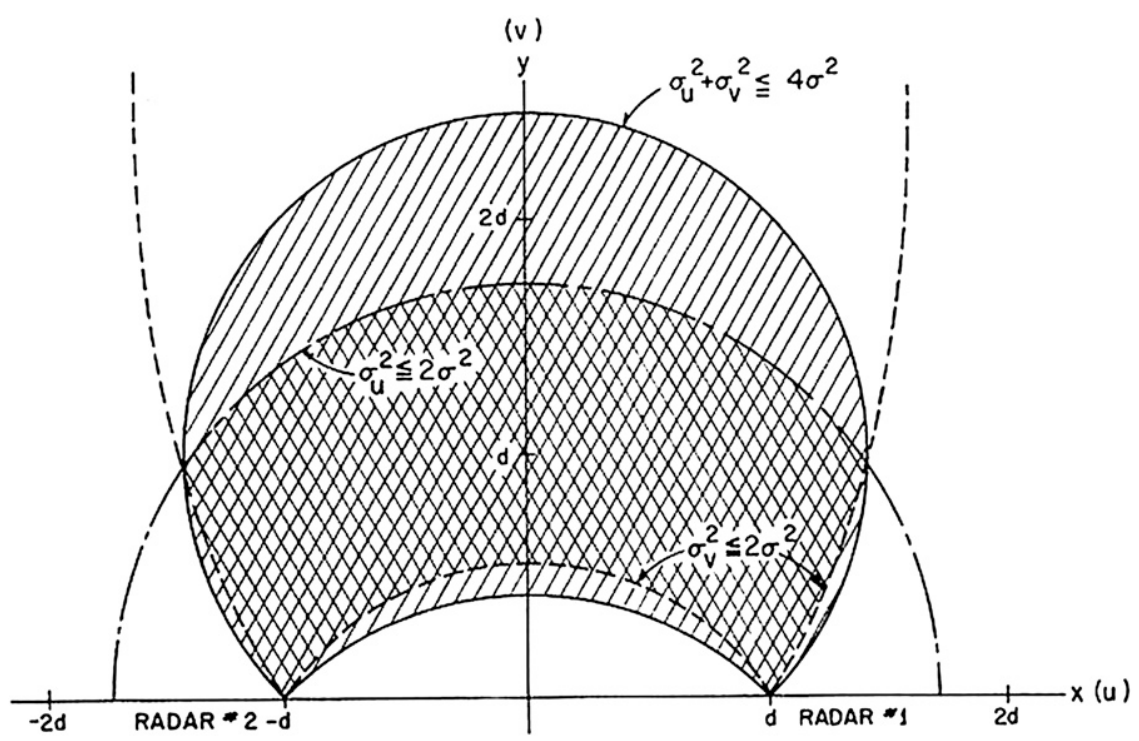

FIG. 12. Dual coplanar Doppler variance diagram showing the tendencies for the uncertainty in $u$ and $v$ components and the combined vector (Lhermitte and Miller 1970).

\section{e. Noise pollution}

Our model was tested numerically with varying levels of independent random noise to synthetically pollute the data, rather than the direct evaluation of uncertainty statistics in each bin. The evaluation of 3D matrices that were a consequence of these statistics caused computer memory issues and became time prohibitive. The radial velocity data were polluted with varying levels of zero-mean Gaussian independent random noise to test the sensitivity of the least squares algorithm with our dataset. Vorticity, swirl, and streamline fields that resulted from data polluted with noise of standard deviations less than $1 \mathrm{~m} \mathrm{~s}^{-1}$ suffered only minor degradation for the results of large-scale streamline circulations and the shape and magnitude of the individual subrotors. Figures 13a-d exhibit this gradual degradation with increasing levels of noise for 1104 PST (1904 UTC), shown in color without noise in Fig. 7. A key result of this exercise is that this least squares technique can handle reasonable levels of random noise in measurement and naturally occurring variations, while still capturing the mean time-spatial phenomena that exist in the flow.

\section{Summary and conclusions}

One major goal of the T-REX field campaign was to characterize the flow conditions leading to the development of rotors and subrotors. Equally important was the characterization of the rotors-subrotors them- selves (i.e., their spatial extent, longevity, strength, evolution, and effect on the MWRBL system). The dual coplanar Doppler lidar measurements and the retrieval methods presented herein provide a unique opportunity to analyze $2 \mathrm{D}$ velocity vectors on an elevated crossbarrier plane. Radial velocity measurements from independent lidars were assembled into velocity vectors using a least squares retrieval method. Note that the use of Taylor's hypothesis was not required and the measurements are obtained in clear air without disturbance of the flow field. This approach has allowed a first look into the character and dynamics of clear air rotors and subrotors at an approximate 130-m resolution. The following flow parameters were calculated: $2 \mathrm{D}$ velocity vectors, vorticity, swirling strength, and velocity streamlines. Their visualization provides evidence of the existence and propagation of two classes of vortical motions: rotors and subrotors. The larger-scale and less coherent vortical motions were described as rotors. The behavior of the attending lee-wave (or gravity current) and the low-level westward moving flow appear to dominate rotor development and evolution. Rotors were oriented with clockwise rotation (positive vorticity) and were often accompanied by strong westerly flow on the lower levels. Constellations of high concentrations of swirl strength and vorticity may exist inside rotors, possibly as a result of the process of rotor genesis or the capturing of advecting vortices by the larger-scale rotor circulation.

Subrotors typically were advected with the mean flow from west to east but were sometimes entrained into a reverse flow. Subrotors were ubiquitous, more intense 

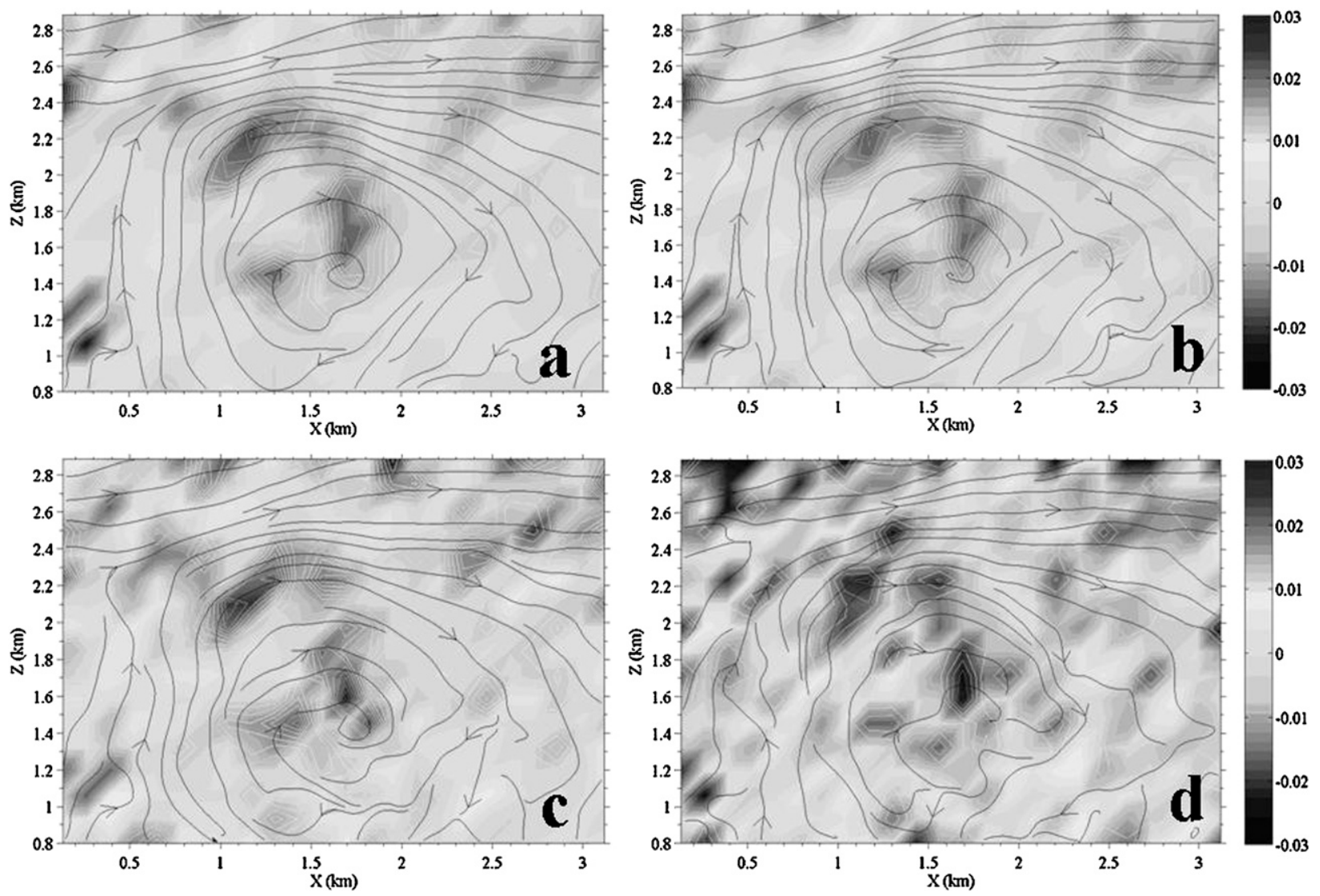

FIG. 13. Vorticity, swirl, and streamline subplots at 1104 PST (1904 UTC) with increasing levels of zero mean Gaussian noise. Mean domain velocity $\langle u\rangle$ is approximately $3.2 \mathrm{~m} \mathrm{~s}^{-1}$. Noise magnitude indicated by standard deviation $\sigma_{\text {Noise }}$ : (a) No noise, (b) $\sigma_{\text {Noise }}=$ $1.00 \mathrm{~m} \mathrm{~s}^{-1}$ and $\sigma_{\text {Noise }} /\langle u\rangle=0.31$, (c) $\sigma_{\text {Noise }}=2.00 \mathrm{~m} \mathrm{~s}^{-1}$ and $\sigma_{\text {Noise }} /\langle u\rangle=0.63$, and (d) $\sigma_{\text {Noise }}=3.00 \mathrm{~m} \mathrm{~s}^{-1}$ and $\sigma_{\text {Noise }} /\langle u\rangle=0.94$.

(in terms of vorticity and swirl) than the full rotors, and were commonly observed traveling on the edges of the main flow. A majority of subrotors at the higher levels rotated clockwise, while some near the ground rotated counterclockwise. At times vortices appeared to be part of a pair (positive and negative). Both rotors and subrotors had maximum swirling strengths and vorticity that usually did not exceed $\pm .03 \mathrm{~s}^{-1}$ in our given time and space window.

The measurement technique could be improved with enhanced lidar synchrony and reduced range gate size, thereby decreasing the sizes of the spatial and temporal grid cells. To achieve this would likely require central control of both lidars with a single computer, clock, and scanning algorithm. Limiting the RHI scanning angles to exclude regions deemed less reliable by the uncertainty scheme analysis would reduce the amount of scanning in ill-conditioned regions. This would also reduce the time between successive lidar scans, improving the temporal resolution. Higher temporal and spatial resolution would likely lead to higher, more instantaneous measurements of vorticity and swirl strength. Additionally, more robust filtering techniques may improve the separation of useful signal from inherently noisy lidar data.

\section{REFERENCES}

Adrian, R. J., K. T. Christensen, and Z.-C. Liu, 2000: Analysis and interpretation of instantaneous turbulent velocity fields. Exp. Fluids, 29, 275-290.

Armijo, L., 1969: A theory for the determination of wind and precipitation velocities with Doppler radars. J. Atmos. Sci., 26, 570-573.

Banta, R. M., L. D. Olivier, W. D. Neff, D. H. Levinson, and D. Ruffieux, 1995: Influence of canyon-induced flows on flow and dispersion over adjacent plains. Theor. Appl. Climatol., 52, 27-42.

,,-- P. H. Gudiksen, and R. Lange, 1996: Implications of small-scale flow features to modeling dispersion over complex terrain. J. Appl. Meteor., 35, 330-342.

—_ and Coauthors, 1997: Nocturnal cleansing flows in a tributary valley. Atmos. Environ., 31, 2147-2162.

Calhoun, R., 1998: Numerical investigations of turbulent flow over complex terrain. Ph.D. dissertation, Stanford University, 278 pp.

_ Neutral case. J. Geophys. Res., 106 (C5), 9277-9293. 
and J. Koseff, 2001: Turbulent flow over a wavy surface: Stratified case. J. Geophys. Res., 106 (C5), 9295-9310.

, R. Heap, M. Princevac, J. Sommer, H. Fernando, and D. Ligon, 2004: Measurement of winds flowing toward an urban area using coherent Doppler lidar. Preprints, Fifth Conf. on the Urban Environment, Vancouver, BC, Canada, Amer. Meteor. Soc., 3.8. [Available online at http://ams.confex.com/ ams/pdfpapers/80377.pdf.]

,,,--- R. Newsom, H. Fernando, and D. Ligon, 2006: Virtual towers using coherent Doppler lidar during the Joint Urban 2003 Dispersion Experiment. J. Appl. Meteor. Climatol., 45, 1116-1126.

Chacín, J., and B. Cantwell, 1997: Study of turbulence structure using the invariants of the velocity gradient tensor. Stanford University Rep. TF-70, 130 pp.

Chong, M. S., A. Perry, and B. Cantwell, 1990: A general classification of three-dimensional flow fields. Phys. Fluids, A2, 765-777.

Collier, C., and Coauthors, 2005: Dual Doppler lidar measurements for improving dispersion models. Bull. Amer. Meteor. Soc., 86, 825-838.

Darby, L., and G. Poulos, 2006: The evolution of lee-wave-rotor activity in the lee of Pike's Peak under the influence of a cold frontal passage: Implications for aircraft safety. Mon. Wea. Rev., 134, 2857-2876.

Davies, F., C. Collier, and K. Bozier, 2005: Errors associated with dualDoppler lidar turbulence measurements. J. Opt., 7A, S280-S289.

Doyle, J., and D. Durran, 2002: The dynamics of mountain-waveinduced rotors. J. Atmos. Sci., 59, 186-201.

- , and 2007: Rotor and subrotor dynamics in the lee of three-dimensional terrain. J. Atmos. Sci., 64, 4202-4221.

Frehlich, R., S. M. Hannon, and S. W. Henderson, 1994: Performance of a $2-\mu \mathrm{m}$ coherent Doppler lidar for wind measurements. J. Atmos. Oceanic Technol., 11, 1517-1528.

Fritsch, F. N., and R. E. Carlson, 1980: Monotone piecewise cubic interpolation. SIAM J. Numer. Anal., 17, 238-246.

Grubišić, V., and J. M. Lewis, 2004: Sierra Wave Project revisited: 50 years later. Bull. Amer. Meteor. Soc., 85, 1127-1142.

—, and M. Orlić, 2007: Early observations of rotor clouds by Andrija Mohorovičić. Bull. Amer. Meteor. Soc., 88, 693-700.

_ J. D. Doyle, J. Kuettner, G. S. Poulos, and C. D. Whiteman, 2004: Terrain-Induced Rotor Experiment: Scientific overview document and experiment design. UCAR, $72 \mathrm{pp}$. [Available online at http://www.eol.ucar.edu/projects/trex/documents/ TREX_SOD.pdf.]

Holmboe, J., and H. Klieforth, 1957: Investigation of mountain lee waves and the air flow over the Sierra Nevada: Final report Department of Meteorology, UCLA, Contract AF 19(604)728, 283 pp.

Hunt, J., A. Wray, and P. Moin, 1988: Eddies, stream, and convergence zones in turbulent flows. Center for Turbulence Research Rep. CTR-S88, 193 pp.
Jeong, J., and F. Hussain, 1995: On the identification of a vortex. J. Fluid Mech., 285, 69-94.

Kuettner, J., 1959: The rotor in the lee of mountains. Geophysics Research Directorate Note 6, AD-208862, Air Force Cambridge Research Laboratories, 20 pp.

Lhermitte, R., and L. Miller, 1970: Doppler radar methodology for the observation of convective storms. Preprints, 14th Radar Meteorology Conf., Tucson, AZ, Amer. Meteor. Soc., 133-138.

Lugt, H., 1979: The dilemma of defining a vortex. Recent Developments in Theoretical and Experimental Fluid Mechanics, U. Müller, K. G. Roesner, and B. Schmidt, Eds., Springer, 309-321.

Lyra, G., 1943: Theorie der stationaeren Leewellenstronung in freier Atmospharere. Z. Angew. Math. Mech., 23, 1-28.

Montgomery, D., G. Runger, and N. Hubele, 2006: Engineering Statistics. John Wiley \& Sons, 457 pp.

National Transportation Safety Board, 2001: Aircraft accident report: Uncontrolled descent and collision with terrain, United Airlines flight 585, Boeing 737-200, N999UA, 4 miles south of Colorado Springs Municipal Airport, Colorado Springs, Colorado, March 3, 1991. NTSB Rep. AAR-01/01, NTIS Rep. PB2001-910401, 15-35.

Newsom, R., R. Calhoun, D. Ligon, and J. Allwine, 2008: Linearly organized turbulence structures observed over a suburban area by dual-Doppler lidar. Bound.-Layer Meteor., 127, 111-130.

Nicholls, J. M., 1973: The airflow over mountains: Research 19581972. WMO Tech. Note 127, World Meteorological Organization, $77 \mathrm{pp}$.

Queney, P., 1955: Rotor phenomena in the lee of mountains. Tellus, 7, 367-371.

— G. Gorby, N. Gerbier, H. Koshmieder, and J. Zierep, 1960: The airflow over mountains. WMO Tech. Note 34, World Meteorological Organization, $135 \mathrm{pp}$.

Ralph, F. M., J. Neiman, T. L. Keller, D. Levinson, and L. Fedor, 1997: Observations, simulations, and analysis of nonstationary trapped lee waves. J. Atmos. Sci., 54, 1308-1333.

Smith, R. B., 1976: Generation of lee waves by the Blue Ridge. J. Atmos. Sci., 33, 507-519.

- 1988: Linear theory of stratified flow past an isolated mountain in isosteric coordinates. J. Atmos. Sci., 45, 3889-3896.

Wolberg, J., 2006: Data Analysis Using the Method of Least Squares: Extracting the Most from Your Experiments. Springer, 44 pp.

Wurtele, M., A. Datta, and R. Sharman, 1993: Lee waves: Benign and malignant. NASA-CR-186024, 26 pp. [Available online at http:// www.nasa.gov/centers/dryden/pdf/88288main_H-1890.pdf.]

Xia, Q., C.-L. Lin, R. Calhoun, and R. Newsom, 2008: Retrieval of urban boundary layer structures from Doppler lidar data. Part I: Accuracy assessment. J. Atmos. Sci., 65, 3-20.

Zhou, J., R. Adrian, S. Balachander, and T. Kendall, 1999: Mechanisms for generating coherent packets of hairpin vortices in channel flow. J. Fluid Mech., 387, 353-396. 